\title{
FRACTAL PROPERTIES OF INVARIANT SUBSETS FOR PIECEWISE MONOTONIC MAPS ON THE INTERVAL
}

\author{
FRANZ HOFBAUER AND MARIUSZ URBAŃSKI
}

\begin{abstract}
Let $T$ be a piecewise monotonic transformation on $[0,1]$ and let $A$ be a $T$-invariant subset, which has positive topological entropy and satisfies the Darboux property. A general existence theorem for conformal measures on $A$ is proved. This is then used to show equality of the dynamical dimension of $A$ and the minimal zero of a certain pressure function.
\end{abstract}

\section{INTRODUCTION}

A map $T:[0,1] \rightarrow[0,1]$ is called piecewise monotonic, if there is a finite subset $\left\{a_{0}, a_{1}, \ldots, a_{N}\right\}$ of $[0,1]$ with $0=a_{0}<a_{1}<\cdots<a_{N}=1$ such that $T \mid\left(a_{i-1}, a_{i}\right)$ is strictly monotone and continuous for $1 \leq i \leq N$. The aim of this paper is to investigate fractal dimensions, pressure functions and conformal measures for invariant subsets of $([0,1], T)$.

The dynamical system $([0,1], T)$ which we consider is allowed to have discontinuities on the set $P:=\left\{a_{0}, a_{1}, \ldots, a_{N}\right\}$ of partition points. If $\lim _{x \uparrow a} T(x)$

$\neq \lim _{x \downarrow a} T(x)$ for some $a \in P$ then there is no natural choice for $T(a)$. Therefore we introduce definitions which do not depend on the values of $T$ at its points of discontinuity. We say that a closed set $A \subset[0,1]$ is $T$-invariant, if $T(A \backslash P) \subset A$. This implies $T(A) \subset A$ if $T$ is continuous. Let $A$ be closed and $T$-invariant. We consider the following definition of conformal measures. Set $\mathscr{Z}=\left\{\left(a_{0}, a_{1}\right),\left(a_{1}, a_{2}\right), \ldots,\left(a_{N-1}, a_{N}\right)\right\}$ and let $g$ be a nonnegative function on $A$. A probability measure $m$ on $A$ is called almost $g$-conformal, if

$$
m(T(Y))=\int_{Y} g d m \text { for all } Y \subset A \text { contained in some } Z \in \mathscr{Z} \text {. }
$$

According to [2], the measure $m$ is called $g$-conformal, if (1.1) holds for all $Y$, for which $T \mid Y$ is strictly monotone. The difference is that $Y$ in (1.1) cannot contain points of $P$. Finally we consider the dynamical dimension as introduced in [ 3 and 4]. The Hausdorff dimension $\operatorname{HD}(\mu)$ of a probability measure $\mu$ is defined as inf $\operatorname{HD}(B)$, where $\operatorname{HD}(B)$ denotes the Hausdorff dimension of the set $B$ and where the infimum is taken over all Borel subsets

Received by the editors July 6, 1992.

1991 Mathematics Subject Classification. Primary 26A18, 54H20; Secondary 28D20.

Research for this paper was done in part, when both authors were visiting the Sonderforschungsbereich 170 at the University of Göttingen and the second author was visiting the University of North Texas in Denton. 
$B$ with $\mu(B)=1$. The dynamical dimension $\operatorname{dD}(A)$ of $A$ is now defined as sup $\operatorname{HD}(\mu)$, the supremum taken over all ergodic invariant measures $\mu$ on $A$ with positive entropy.

The first aim of this paper is to give a version of the definition of pressure on $A$ for a class of piecewise continuous functions $f$, denoted by $q(T \mid A, f)$, such that the minimum $z_{A}$ of zeros of $t \mapsto q\left(T|A,-t \log | T^{\prime} \mid\right)$ exists, and to show existence of almost $e^{q(T \mid A, f)-f}$-conformal measures. These are then the prerequisites for the second aim of this paper, which is to show equality of $z_{A}$, of $\mathrm{dD}(A)$ and of the infimum of all $t$, for which an almost $\left|T^{\prime}\right|^{t}$-conformal measure on $A$, whose support equals $A$, exists. The reader should think of these three equalities as a version of the McCluskey-Manning-Bowen formula stated for maps of the interval. He should be also aware that our real aim would be to have this formula for $\operatorname{HD}(A)$ instead of $\operatorname{dD}(A)$. Under certain additional assumptions on $T$ like $\inf \left|T^{\prime}\right|>1$ this is done in [13 and 8]. However the problem, whether $\operatorname{HD}(A)$ equals $\mathrm{dD}(A)$ in general, seems to be very difficult and so far is open. Nevertheless the partial result proved in our paper seems to be also of interest. For an additional discussion about pressure, Hausdorff dimension and conformal measures the reader is referred to [3, 4, and 8].

In order to prove such results we need several assumptions on $T$ and on $A$. As in [8], an open nonempty interval $I \subset[0,1]$ is called atomic, if $T^{n}(I)$ is contained in some element of $\mathscr{Z}$ for all $n \geq 0$. We say that the piecewise monotonic map $T$ is regular, if there are no atomic intervals, or if there are finitely many intervals $I_{1}, I_{2}, \ldots, I_{l}$, mapped monotonically to each other by $T$, such that for each atomic interval $I$ there is an $n$ and a $k$ with $T^{n}(I) \subset I_{k}$. It is well known that all unimodal maps without stable periodic orbits and with negative Schwarzian derivative are regular. Much weaker conditions, obtained recently, for a piecewise monotonic map to be regular can be found for example in [11] or [12]. Furthermore, we say that $T$ is piecewise differentiable, if the derivative of $T \mid Z$ exists for all $Z \in \mathscr{Z}$ and can be extended to a continuous function on the closure of $Z$.

A closed subset $A$ of $([0,1], T)$ is called a Darboux subset, if it satisfies $T(A \backslash P) \subset A$, if it is disjoint from atomic intervals and if the following holds

$$
T(A \cap Z)=A \cap T(Z) \text { for all } Z \in \mathscr{Z}
$$

which sometimes is called Darboux property.

Darboux subsets occur in a natural way. Let $T$ be a regular piecewise monotonic map. Let $G \subset[0,1]$ be an invariant set which is a finite union of intervals and which contains the intervals attracting all atomic intervals. Set $A=\bigcap_{i=0}^{\infty} \overline{[0,1] \backslash T^{-i}(G)}$. Its complement is the set of all $x$, for which there is a neighbourhood $U$ and an $i \geq 0$ with $T^{i}(U) \subset G$. Clearly $A$ is closed, has empty intersection with all atomic intervals and satisfies $T(A \backslash P) \subset A$. Furthermore, if $x \in A \cap T(Z)$ for some $Z \in \mathscr{Z}$, then $(T \mid Z)^{-1}(x) \in Z$ and also $(T \mid Z)^{-1}(x) \in A$ by definition of $A$. Hence $x \in T(A \cap Z)$, which implies the nonobvious part of (1.2). Therefore $A$ is a Darboux subset. In [6], a decomposition of the nonwandering set of a piecewise monotonic map into topologically transitive components is given. Each component in this decomposition, which has positive topological entropy, is of the above form and hence a Darboux subset.

Throughout the paper we shall consider a regular piecewise monotonic map 
$T$, which is piecewise differentiable and a Darboux subset $A$ of $([0,1], T)$ with $h_{\text {top }}(T \mid A)>0$. In $\S 2$ we develop a definition $q(T \mid A, f)$ of the pressure for a class $D(A)$ of piecewise continuous functions $f$ containing $h:=$ $-\log \left|T^{\prime}\right|$, if $T^{\prime} \mid Z$ is nonzero for all $Z \in \mathscr{Z}$. We show that the map $t \mapsto$ $q(T \mid A, t h)$ has a minimal zero $z_{A}$ in $[0,1]$. The definition of $q(T \mid A, f)$ is based on an approximation of $(A, T \mid A)$ from below by dynamical systems, which behave like subshifts of finite type. This technique of approximation is studied further in $\S 3$ and used then in $\S 4$ to show existence of almost $e^{q(T \mid A, f)-f}$-conformal measures on $A$ for $f \in D(A)$. We give also conditions, under which conformal measures exist.

In $\S 5$ we assume additionally that $T^{\prime} \mid Z$ is nonzero and of bounded variation for each $Z \in \mathscr{Z}$, and that $A$ is topologically transitive. Let $\delta(A)$ be the infimum of all $t$ for which an almost $\left|T^{\prime}\right|^{t}$-conformal measure exists on $A$, whose support equals $A$. Using the existence theorem for almost conformal measures of $\S 4$ and results of [10], we show that $\mathrm{dD}(A)=z_{A}=\delta(A)$.

\section{Pressure}

In this section we develop a version of the definition of pressure, which is suitable for later applications. Let $T$ be a regular piecewise monotonic map and let $A$ be a Darboux subset of $([0,1], T)$. Let $D(A)$ be the set of all $f: A \rightarrow(-\infty, \infty]$, such that $f \mid A \cap Z$ has values in $\mathbb{R}$ and can be extended to a continuous function from the closure of $A \cap Z$ to $(-\infty, \infty]$ for all $Z \in \mathscr{Z}$, where we use the obvious topology on $(-\infty, \infty]$.

We introduce a definition of invariant measures which avoids discontinuities of the considered mappings. For a closed invariant subset $B$ of $[0,1]$ let $M_{T}(B)$ be the set of all $T$-invariant probability measures on $B$, for which the set of discontinuities of $T$ is of measure zero, and let $M_{T}(B, f)$ be the set of those measures in $M_{T}(B)$ for which the set of discontinuities of $f$ is a nullset. Remark that these sets of discontinuities are contained in the set $P$ of partition points and hence are finite. Furthermore, let $E_{T}(B)$ and $E_{T}(B, f)$ denote the sets of ergodic measures in $M_{T}(B)$ and in $M_{T}(B, f)$ respectively. For continuous $T$ and $f$ we get the usual definitions. If $f \in D(A)$, then $f$ is bounded from below and hence $\int f d \mu$ exists for $\mu \in M_{T}(A)$ allowing that $\int f d \mu=\infty$.

The first step in the definition of pressure is to cut out partition points $a \in$ $P$ with infinite orbit. To this end set $T^{+}(a)=\lim _{x \downarrow a} T(x)$ and $T^{-}(a)=$ $\lim _{x \uparrow a} T(x)$. Furthermore define $N^{+}=\left\{a \in P: T^{i}\left(T^{+}(a)\right) \notin P\right.$ for $\left.i \geq 0\right\}$ and $N^{-}=\left\{a \in P: T^{i}\left(T^{-}(a)\right) \notin P\right.$ for $\left.i \geq 0\right\}$. Then let $P^{+}$be the set of elements of $N^{+}$for which $T^{+}(a)$ has an infinite orbit. Similarly define $P^{-}$. Let $\mathscr{X}$ denote the family of all open subsets of $[0,1]$, which contain a neighbourhood of each $a \in P^{+} \cap P^{-}$and which contain an interval $(a-\varepsilon, a)$ or $(a, a+\varepsilon)$ for some $\varepsilon>0$, if $a \in P^{-} \backslash P^{+}$or if $a \in P^{+} \backslash P^{-}$respectively. If $T$ is continuous on $[0,1]$ then $P^{+}=P^{-}$and the elements of $\mathscr{X}$ are just neighbourhoods of $P^{+}=P^{-}$. Now let $\left(V_{n}\right)_{n \geq 1}$ be a decreasing sequence in $\mathscr{Z}$ satisfying $\bigcap_{n=1}^{\infty} V_{n}=P^{+} \cap P^{-}$. Set $A_{n}=\left\{x \in A: T^{i}(x) \notin V_{n}\right.$ for $\left.i \geq 1\right\}$. It may happen that $A_{n}=\varnothing$. But if $A$ contains a periodic orbit, which is disjoint from $P$, then $A_{n} \neq \varnothing$ for all large $n$. It is shown in $\S 3$ that this always happens, if $h_{\text {top }}(A)>0$. For $f \in D(A)$ set $f_{n}=\min (f, n)$, which is again in 
$D(A)$. We define

$$
q\left(T \mid A_{n}, f_{n}\right)=\sup _{\mu \in E_{T}\left(A_{n}, f_{n}\right)} h_{\mu}+\int f_{n} d \mu .
$$

If $T \mid A_{n}$ and $f_{n}$ are continuous, the variational principle implies that $q\left(T \mid A_{n}, f_{n}\right)$ coincides with the usual definition of pressure (see [14]). Since the sequences $\left(A_{n}\right)_{n \geq 1}$ and $\left(f_{n}\right)_{n \geq 1}$ are increasing also $\left(q\left(T \mid A_{n}, f_{n}\right)\right)_{n \geq 1}$ is increasing and we can define

$$
q(T \mid A, f)=\sup _{n \geq 1} q\left(T \mid A_{n}, f_{n}\right)=\lim _{n \rightarrow \infty} q\left(T \mid A_{n}, f_{n}\right)
$$

which is the notion of pressure we use in this paper. The following lemma shows that $q(T \mid A, f)$ does not depend on the sequences $\left(A_{n}\right)_{n \geq 1}$ and $\left(f_{n}\right)_{n \geq 1}$ occurring in its definition.

Lemma 1. For $f \in D(A)$ let $\widetilde{E}_{T}(A, f)$ be the set of all $\mu \in E_{T}(A, f)$, such that each $a \in P^{+}$is not a limit point from the right of $\operatorname{supp} \mu$ and such that each $a \in P^{-}$is not a limit point from the left of $\operatorname{supp} \mu$. Then

$$
q(T \mid A, f)=\sup _{\mu \in \widetilde{E}_{T}(A, f)} h_{\mu}+\int f d \mu .
$$

Proof. Let $\left(A_{n}\right)_{n \geq 1}$ and $\left(f_{n}\right)_{n \geq 1}$ be as in the definition of $q(T \mid A, f)$. If $\mu \in \widetilde{E}_{T}(A, f)$, then there is a $k$ with $\operatorname{supp} \mu \subset A_{n}$ for $n \geq k$ be definition of $\widetilde{E}_{T}(A, f)$. If $n$ is large enough, the points of discontinuity of $f$ and $f_{n}$ are the same, and we get $\mu \in E_{T}\left(A_{n}, f_{n}\right)$. Hence we get from (2.1) that $q(T \mid A, f) \geq \lim _{n \rightarrow \infty} h_{\mu}+\int f_{n} d \mu$, which equals $h_{\mu}+\int f d \mu$ by monotone convergence. On the other hand, for any $r<q(T \mid A, f)$, there is an $n$ with $q\left(T \mid A_{n}, f_{n}\right)>r$. By $(2.1)$ there is a $\mu \in E_{T}\left(A_{n}, f_{n}\right)$ with $h_{\mu}+\int f_{n} d \mu>r$. Since $f \geq f_{n}$, we get $h_{\mu}+\int f d \mu>r$. Since $f \geq f_{n}$ have the same points of discontinuity, if $n$ is large enough, we get $\mu \in E_{T}\left(A_{n}, f\right)$ for all large $n$. The definition of $A_{n}$ implies that $E_{T}\left(A_{n}, f\right) \subset \widetilde{E}_{T}(A, f)$, which finishes the proof.

We give a first application. We denote by $h$ the restriction of the function $-\log \left|T^{\prime}\right|$ to $A$. If $T$ is piecewise differentiable and if all zeros of $T^{\prime}$ are in $P$, then $h \in D(A)$. For $t \geq 0$ we can define

$$
q(t)=q(T \mid A, t h) .
$$

The proof of the next theorem uses results of $\S 3$, which are completely independent of this section. If $T$ has discontinuities, then $h_{\text {top }}(T \mid A)$ can be defined as $\sup _{M_{T}(A)} h_{\mu}$.

Theorem 1. Let $T$ be a regular piecewise monotonic map, which is piecewise differentiable and whose derivative is nonzero on $[0,1] \backslash P$. If $A$ is a Darboux subset of $([0,1], T)$ with $h_{\mathrm{top}}(T \mid A)>0$, then $q:[0,1] \rightarrow(-\infty, \infty]$ has the following properties.

(i) $q(0) \geq 0$ and $q(1) \leq 0$,

(ii) $q$ is convex and hence continuous,

(iii) $0<t_{1}<t_{2} \leq 1 \Rightarrow q\left(t_{1}\right) \geq q\left(t_{2}\right)$.

Proof. Lemma 5 of the next section shows that $A_{n} \neq \varnothing$ for all large $n$. Hence (2.1) and (2.2) imply that $q(0)=q(T \mid A, 0) \geq 0$. If $q(1)>0$, then Lemma 
1 implies that there is a $\mu \in \widetilde{E}_{T}(A, h)$ with $h_{\mu}+\int h d \mu>0$. By (ii) of Lemma 6 we get $h_{\mu}>0$. Now Theorem 2 of [7] implies that $h_{\mu} \leq-\int h d \mu$, a contradiction, finishing the proof of (i). By (2.1), the function $t \mapsto q\left(T \mid A_{n}, t h_{n}\right)$ is a supremum of linear functions and therefore convex. By (2.2), the function $t \mapsto q(t)$ is a supremum of convex functions and therefore convex. Since every convex function is continuous, (ii) is shown. Suppose that $t_{1}<t_{2}$ and that $q\left(t_{1}\right)<q\left(t_{2}\right)$. By Lemma 1 , there is a $\mu \in \widetilde{E}_{T}(A, h)$ with $h_{\mu}+t_{1} \int h d \mu<$ $h_{\mu}+t_{2} \int h d \mu$. Remark that $\widetilde{E}_{T}(A, t h)$ does not depend on $t$ for $t>0$. We get $t_{1} \int h d \mu<t_{2} \int h d \mu$ and hence $\int h d \mu>0$. This contradicts (ii) of Lemma 6 showing (iii).

Theorem 1 implies that $q$ is bounded on $[0,1]$ and that there is a unique $z_{A} \in[0,1]$ with $q\left(z_{A}\right)=0$, such that $q(t)>0$ for $t \in\left[0, z_{A}\right)$ and $q(t) \leq 0$ for $t \in\left[z_{A}, 1\right]$.

\section{APPROXIMATION WITH SUBSHIFTS OF FINITE TYPE}

In this section we again assume that $T$ is a regular piecewise monotonic map and that $A$ is a Darboux subset of $([0,1], T)$. We exhaust $(A, T \mid A)$ by subshifts of finite type. To this end we have to modify $([0,1], T)$ refining the partition given by $P$ and doubling all inverse images of the new partition points.

We use the sets $N^{+}, N^{-}, P^{+}, P^{-}$defined in $\S 2$. If $a \in P \backslash N^{+}$then there is a minimal $j(a)>0$ with $T^{j(a)}\left(T^{+}(a)\right) \in P$. Set $Q_{1}^{+}=\left\{T^{i}\left(T^{+}(a)\right): a \in\right.$ $\left.P \backslash N^{+}, 0 \leq i<j(a)\right\}$. If $a \in N^{+} \backslash P^{+}$, the orbit of $T^{+}(a)$ is finite. Set $Q_{2}^{+}=\left\{T^{i}\left(T^{+}(a)\right): a \in N^{+} \backslash P^{+}, i \geq 0\right\}$. In the same way define $Q_{1}^{-}$and $Q_{2}^{-}$. Since $T$ is regular, there are finitely many intervals $I_{1}, I_{2}, \ldots, I_{l}$, mapped monotonically to each other, which attract all atomic intervals. Since $T$ induces a permutation of these intervals, there is a set $Q_{3}$ consisting of finitely many periodic orbits, such that the closure of each $I_{j}$ has nonempty intersection with $Q_{3}$. Set $Q=P \cup Q_{1}^{+} \cup Q_{1}^{-} \cup Q_{2}^{+} \cup Q_{2}^{-} \cup Q_{3}$. This set is finite and considered as the set of new partition points. It is chosen such that

$$
a \in Q \backslash P^{+} \Leftrightarrow T^{+}(a) \in Q \quad \text { and } \quad a \in Q \backslash P^{-} \Leftrightarrow T^{-}(a) \in Q .
$$

Set $R=\left\{x \in(0,1): T^{i}(x) \in Q\right.$ for some $\left.i \geq 0\right\}$. In the interval $[0,1]$ substitute each $x$ by two point $x^{+}$and $x^{-}$, which gives a totally ordered compact set $X$. Consider $(0,1) \backslash R$, which is dense in $X$. As $T$ is piecewise monotonic with discontinuities in $P \subset Q$, we can extend $T$ continuously from $(0,1) \backslash R$ to all of $X$ and get a continuous map $\widetilde{T}: X \rightarrow X$. Define $\pi: X \rightarrow$ $[0,1]$ by $\pi(x)=x$ if $x \notin R$ and by $\pi\left(x^{+}\right)=\pi\left(x^{-}\right)=x$ if $x \in R$. Then $\pi$ is continuous and

$$
T(\pi(x))=\pi(\widetilde{T}(x)) \text { if } T \text { is continuous in } \pi(x) .
$$

If $Q=\left\{b_{0}, b_{1}, \ldots, b_{K}\right\}$ and $0=b_{0}<b_{1}<\cdots<b_{K}=1$, set

$$
\mathscr{U}=\left\{\left[0, b_{1}^{-}\right],\left[b_{1}^{+}, b_{2}^{-}\right], \ldots,\left[b_{K-1}^{+}, 1\right]\right\}
$$

which is a partition of $X$ into closed-open intervals, on each of which $\widetilde{T}$ is strictly monotone. 
The set $A^{\prime}:=A \cap((0,1) \backslash R)$ is contained in $(0,1) \backslash R \subset X$ and satisfies $T\left(A^{\prime}\right) \subset A^{\prime}$, since $T(A \backslash P) \subset A$ and $T^{-1}(R) \subset R$. Let $\widetilde{A}$ be the closure of $A^{\prime}$ in $X$. Since $\widetilde{T}$ is continuous, we get $\widetilde{T}(\widetilde{A}) \subset \widetilde{A}$ and $\pi(\widetilde{A})=A$. For $f \in D(A)$ let $\tilde{f}$ be the continuous extension of $f \mid A^{\prime}$ to $\tilde{A}$. Then $\tilde{f}: \tilde{A} \rightarrow(-\infty, \infty]$ is continuous and

$$
f(\pi(x))=\tilde{f}(x) \text { if } f \text { is continuous in } \pi(x) .
$$

Lemma 3. If $A$ is a Darboux subset of $([0,1], T)$, then $\mathscr{U}$ is a generator for $\widetilde{A}$, which means the following. If $x, y \in \widetilde{A}$ with $x \neq y$, then there is an $i$ such that $\widetilde{T}^{i}(x)$ and $\widetilde{T}^{i}(y)$ are in different elements of $\mathscr{U}$.

Proof. Fix $x, y \in A$ with $x \neq y$. If $\pi(x)=\pi(y)$, then $T^{j}(\pi(x))=: z \in Q$ for some $j$. Furthermore, $\widetilde{T}^{j}(x)=z^{+}$and $\widetilde{T}^{j}(y)=z^{-}$(or vice versa). This says that $\widetilde{T}^{j}(x)$ and $\widetilde{T}^{j}(y)$ are in different elements of $\mathscr{U}$. Hence suppose $\pi(x) \neq \pi(y)$. Let $J$ be the open interval in $[0,1]$ with endpoints $\pi(x)$ and $\pi(y)$. If $J$ is not atomic, then there is a minimal $j$ with $T^{j}(J) \cap P \neq \varnothing$, which implies that $\widetilde{T}^{j}(x)$ and $\widetilde{T}^{j}(y)$ are in different elements of $\mathscr{U}$. If $J$ is atomic, then $T^{j}(J) \subset I_{k}$ for some $j \geq 0$ and one of the intervals $I_{1}, I_{2}, \ldots, I_{l}$ introduced above. Since $\pi(\widetilde{A})=A$ and $A \cap I_{k}=\varnothing$, the points $\pi\left(\widetilde{T}^{j}(x)\right)$ and $\pi\left(\widetilde{T}^{j}(y)\right)$ are the endpoints of $I_{k}$. Since the closure $\bar{I}_{k}$ of $I_{k}$ contains a point $q$ of $Q_{3} \subset Q$, and since $\widetilde{T}^{j}(x)$ and $\widetilde{T}^{j}(y)$ are limit points of $\widetilde{A} \backslash \pi^{-1}\left(\bar{I}_{k}\right)$, it follows that either $q^{+}$or $q^{-}$is between $\widetilde{T}^{j}(x)$ and $\widetilde{T}^{j}(y)$ which are therefore in different elements of $\mathscr{U}$.

Set $\tilde{Q}=\pi^{-1}(Q)$ and $\widetilde{S}=\widetilde{Q} \backslash \widetilde{T}^{-1}(\widetilde{Q})$. Furthermore, let $\widetilde{S}^{+}$be the set of all elements of $\widetilde{S}$ which are left endpoints of intervals in $\mathscr{U}$ and let $\widetilde{S}^{-}$be the set of all elements of $\widetilde{S}$ which are right endpoints of intervals in $\mathscr{U}$. Now (3.1) implies

$$
s \in \widetilde{S}^{+} \Leftrightarrow \pi(s) \in P^{+} \quad \text { and } \quad s \in \widetilde{S}^{-} \Leftrightarrow \pi(s) \in P^{-} .
$$

This gives

$$
s \in \widetilde{S} \Rightarrow\left\{\widetilde{T}^{i}(s): i \geq 0\right\} \text { is infinite }
$$

since $\widetilde{T}\left|\left(X \backslash \pi^{-1}(R)\right)=T\right|([0,1] \backslash R)$ and since $\widetilde{T}^{i}(\widetilde{S}) \cap \widetilde{Q}=\varnothing$ for all $i \geq 1$.

Now we construct approximations of $\tilde{A}$. Set

$$
\mathscr{U}_{n}=\bigvee_{i=0}^{n-1} T^{-i \mathscr{U}}=\left\{\bigcap_{i=0}^{n-1} T^{-i}\left(U_{i}\right): U_{i} \in \mathscr{U}\right\},
$$

a partition of $X$ into closed-open intervals, on each of which $\widetilde{T}^{n}$ is monotone. For $x \in X$ let $U_{n}(x)$ be the unique element of $\mathscr{U}_{n}$ containing $x$. Set $\widetilde{V}_{n}=$ $\bigcup_{s \in \tilde{S}} U_{n}(s)$ and $\widetilde{A}_{n}=\left\{x \in \widetilde{A}: \widetilde{T}^{i}(x) \notin \widetilde{V}_{n}\right.$ for $\left.i \geq 0\right\}$. Clearly $\widetilde{A}_{n}$ is closed and $\tilde{T}\left(\tilde{A_{n}}\right) \subset \tilde{A}_{n}$.

Lemma 4. Let $A$ be a Darboux subset of $([0,1], T)$.

(i) If $U \in \mathscr{U}_{n}$ and $U \cap \widetilde{S}=\varnothing$ then $\widetilde{T}(U) \in \mathscr{U}_{n-1}$, where $n \geq 2$.

(ii) If $U \in \mathscr{U}_{k}$ for $k \geq 2$ and $U \cap \widetilde{S}=\varnothing$ then $\widetilde{T}(U \cap \widetilde{A})=\tilde{T}(U) \cap \tilde{A}$. If $n \geq k$ then we have also $\widetilde{T}\left(U \cap \widetilde{A}_{n}\right)=\widetilde{T}(U) \cap \widetilde{A}_{n}$. 
(iii) The dynamical system $\left(\tilde{A}_{n}, \widetilde{T} \mid \tilde{A}_{n}\right)$ is topologically conjugate to a subshift of finite type with a finite alphabet, if $n \geq 2$. In particular, $\widetilde{T} \mid \tilde{A}_{n}$ is an open, expansive map.

Proof. If $U \in \mathscr{U}_{n}$ then there are $U_{1} \in \mathscr{U}$ and $U_{2} \in \mathscr{U}_{n-1}$ with $U=U_{1} \cap$ $\widetilde{T}^{-1}\left(U_{2}\right)$. We have $\widetilde{T}(U)=\widetilde{T}\left(U_{1}\right) \cap U_{2}$. We show $U_{2} \subset \widetilde{T}\left(U_{1}\right)$. This is clear if $U$ has no common endpoint with $U_{1}$. If $s$ is a common endpoint of $U$ and $U_{1}$ then $s \in \widetilde{Q} \backslash \widetilde{S}$ by assumption. By definition of $\widetilde{S}$, the common endpoint $\widetilde{T}(s)$ of $\widetilde{T}(U)$ and $\widetilde{T}\left(U_{1}\right)$ is again in $\widetilde{Q}$. Since $\widetilde{T}\left(U_{1}\right) \cap U_{2} \neq \varnothing$ and since $U_{2}$ can contain elements of $\widetilde{Q}$ only as endpoints, it follows that $U_{2}$ is at the same side of $\widetilde{T}(s)$ as $\widetilde{T}\left(U_{1}\right)$. This proves $U_{2} \subset \widetilde{T}\left(U_{1}\right)$ and (i) follows.

Since $\widetilde{T}(U \cap \widetilde{B}) \subset \widetilde{T}(U) \cap \widetilde{B}$ holds for each $\widetilde{B} \subset X$ satisfying $\widetilde{T}(\widetilde{B}) \subset \widetilde{B}$, for (ii) it suffices to show the other inclusion. Fix $y \in \widetilde{T}(U) \cap \widetilde{A}$. By definition of $\tilde{A}$, there are $y_{n} \in A \backslash R$ with $y_{n} \rightarrow y$ in $X$. Since $\widetilde{T}(U)$ is open by (i), we can choose $y_{n} \in \widetilde{T}(U)$. Furthermore, we can assume $y_{n} \notin T(R)$, since $T(R) \backslash R$ is finite. Let $Z \in \mathscr{Z}$ be such that $Z$ contains the interior of $\pi(U)$. Then $y_{n} \in T(Z)$ for $n \geq 1$. Set $x_{n}=(T \mid Z)^{-1}\left(y_{n}\right) \in Z$. Since $T \mid Z$ is strictly monotone, we get from (1.2) that $x_{n} \in A$. Since $y_{n} \notin T(R)$ we get $x_{n} \notin R$. Let $x$ be a limit point of $\left(x_{n}\right)_{n \geq 1}$ in $X$. Then $x \in \tilde{A}$ and $(\widetilde{T} \mid U)^{-1}(y)=x$. Hence $x \in \tilde{A} \cap U$, which means that $y \in T(\tilde{A} \cap U)$ and the first part of (ii) is shown. In order to show the second part, suppose that $y \in \widetilde{T}(U) \cap \widetilde{A}_{n}$. Then $y \in \widetilde{T}(U) \cap \widetilde{A}$. By the first part of (ii) there is an $x \in \widetilde{A} \cap U$ with $\widetilde{T}(x)=y$. Since $U \in \mathscr{U}_{k}$ with $U \cap \widetilde{S}=\varnothing$ and since $n \geq k$ we get that $U \cap \widetilde{V}_{n}=\varnothing$. Therefore $x \notin \widetilde{V}_{n}$. Also $\widetilde{T}^{i}(x)=\widetilde{T}^{i-1}(y) \notin \widetilde{\widetilde{V}}_{n}$ for $i \geq 1$, as $y \in \widetilde{A}_{n}$. We have shown $x \in \widetilde{A}_{n}$. As $\widetilde{T}(x)=y$ and as $x \in U \cap \widetilde{A}_{n}$ we get $y \in \widetilde{T}\left(U \cap \widetilde{A_{n}}\right)$ finishing the proof of (ii).

Set $\mathscr{R}=\left\{U \cap \widetilde{A}_{n} \neq \varnothing: U \in \mathscr{U}_{n}\right\}$, a finite partition of $\widetilde{A}_{n}$ into closedopen sets. If $U \in \mathscr{U}_{n}$ has nonempty intersection with $\widetilde{A}_{n}$, then $U \cap \widetilde{S}=\varnothing$ by definition of $\widetilde{V}_{n}$. By (i) we get $\widetilde{T}(U) \in \mathscr{U}_{n-1}$ and by (ii) we get that $\widetilde{T}\left(U \cap \widetilde{A}_{n}\right)=$ $\widetilde{T}(U) \cap \widetilde{A}_{n}$. This shows that $\widetilde{T}\left(U \cap \widetilde{A}_{n}\right)$ is a finite union of elements of $\mathscr{R}$. Hence the partition $\mathscr{R}$ has the Markov property and (iii) follows.

Lemma 5. Let $A$ be a Darboux subset of $([0,1], T)$.

(i) Then $\widetilde{T}(U \cap \widetilde{A})$ is an interval in $\widetilde{A}$ for all $U \in \mathscr{U}$.

(ii) If $h_{\mathrm{top}}(A)>0$ then $A$ contains a periodic orbit which is disjoint from $P$.

Proof. Fix $U \in \mathscr{U}$. Then $U \backslash \widetilde{S}$ is a union of finitely or countably many disjoint intervals $V$ satisfying $\widetilde{T}(V \cap \widetilde{A})=\widetilde{T}(V) \cap \widetilde{A}$ by (ii) of Lemma 4 . Since $\widetilde{T} \mid U$ is strictly monotone, we get that $\widetilde{T}((U \backslash \widetilde{S}) \cap \widetilde{A})=\widetilde{T}(U \backslash \widetilde{S}) \cap \widetilde{A}$. As $\widetilde{S}$ can contain only endpoints of $U$, this implies that $\widetilde{T}(U \cap \widetilde{A})$ is an interval in $\widetilde{A}$ proving (i).

In the notation of [6], (i) says that $(\tilde{A}, \widetilde{T} \mid \tilde{A})$ is a piecewise monotonic dynamical system. As $\pi$ is bijective except on a countable set, we get $h_{\text {top }}(\widetilde{T} \mid \tilde{A})=$ $h_{\text {top }}(T \mid A)>0$. The results of [6] imply that $\tilde{A}$ contains a topologically transitive subset $\widetilde{L}$ with $h_{\text {top }}(\widetilde{T} \mid \tilde{L})=h_{\text {top }}(\widetilde{T} \mid \widetilde{A})$, which contains a dense set of periodic orbits. This set of periodic orbits has to be infinite and hence there is 
a periodic orbit in $\tilde{A}$ disjoint from $\pi^{-1}(P)$. Its image under $\pi$ is a periodic orbit in $A$, which is disjoint from $P$.

Now we compare the construction of this section with that in $\S 2$.

Lemma 6. Let $A$ be a Darboux subset of $([0,1], T)$. For $f \in D(A)$ let $\tilde{f}$ be the continuous extension of $f \mid A^{\prime}$ to $\tilde{A}$. Furthermore let $p$ denote the pressure of a continuous function on a topological dynamical system.

(i) If $f \in D(A)$ then $q(T \mid A, f)=\lim _{n \rightarrow \infty} p\left(\widetilde{T} \mid \tilde{A}_{n}, \tilde{f}_{n}\right)$, where $\tilde{f}_{n}=$ $\min (n, \tilde{f})$.

(ii) Let $T$ be piecewise differentiable with $T^{\prime} \neq 0$ on $[0,1] \backslash P$. Set $h=$ $-\log \left|T^{\prime}\right|$ on $A$. Then $\int h d \mu \leq 0$ for $\mu \in \widetilde{E}_{T}(A, h)$.

Proof. Let $\widetilde{V}_{n}$ be as in the definition of $\tilde{A}_{n}$. Let $V_{n} \subset[0,1]$ be the interior of $\pi\left(\tilde{V}_{n}\right)$. It follows from (3.4) that $V_{n} \in \mathscr{X}$. By Lemma 3 we get $\bigcap_{n=1}^{\infty} V_{n}=$ $P^{+} \cap P^{-}$. Set $A_{n}=\left\{x \in A: T^{i}(x) \notin V_{n}\right.$ for $\left.i \geq 0\right\}$. First we compare $E_{T}\left(A_{n}\right)$ with the set $E_{\widetilde{T}}\left(\tilde{A}_{n}\right)$ of all $\widetilde{T}$-invariant measures on $\widetilde{A}_{n}$. We show first

$$
\text { for each } \mu \in E_{T}\left(A_{n}\right) \text { there is a } \tilde{\mu} \in E_{\widetilde{T}}\left(\tilde{A}_{n}\right)
$$

$$
\text { with } \tilde{\mu} \circ \pi^{-1}=\mu \text { and } h_{\tilde{\mu}}=h_{\mu} \text {. }
$$

If $\mu \in E_{T}\left(A_{n}\right)$ and $\mu(R)=0$ then set $\tilde{\mu}=\mu \circ \pi$. Since $\tilde{\mu}\left(\pi^{-1}(R)\right)=\mu(R)=0$, $\pi$ is a bijection almost everywhere and (3.6) is shown. If $\mu(R)>0$, then $\mu$ is concentrated on a periodic orbit $L \subset R \cap A_{n}$ and $T$ is continuous on $L$. There are two copies $L^{+}$and $L^{-}$of $L$ in $X$ with $\pi\left(L^{+}\right)=\pi\left(L^{-}\right)=L$. At least one of $L^{+}$and $L^{-}$is in $\widetilde{A}_{n}$. Let $\tilde{\mu}$ be the measure in $E_{\widetilde{T}}\left(\widetilde{A}_{n}\right)$ concentrated on it and (3.6) holds by (3.2).

Now let $N$ be the set of all $\tilde{\mu} \in E_{\widetilde{T}}\left(\tilde{A}_{n}\right)$ with $\tilde{\mu}(\tilde{Q})>0$. As $\tilde{Q}$ is finite, also $N$ is finite. If $\tilde{\mu} \in E_{\widetilde{T}}\left(\tilde{A}_{n}\right) \backslash N$, then $\tilde{\mu}\left(\pi^{-1}(R)\right)=0$. Set $\mu=\tilde{\mu} \circ \pi^{-1}$. Then $\mu(R)=0$ and $\pi$ is a bijection almost everywhere. Since discontinuities of $T$ and $f \in D(A)$ are contained in $P \subset R$, we get $\mu \in E_{T}\left(A_{n}, f\right)$. We have shown

$$
\tilde{\mu} \in E_{\widetilde{T}}\left(\tilde{A}_{n}\right) \backslash N \Rightarrow \mu:=\tilde{\mu} \circ \pi^{-1} \in \bigcap_{f \in D(A)} E_{T}\left(A_{n}, f\right) \text { and } h_{\tilde{\mu}}=h_{\mu} .
$$

Let $F_{\widetilde{T}}\left(\widetilde{A_{n}}\right)$ be the set of all measures in $E_{\widetilde{T}}\left(\widetilde{A}_{n}\right)$ concentrated on periodic orbits. Then

$$
\tilde{\mu} \in E_{\widetilde{T}}\left(\tilde{A}_{n}\right) \Rightarrow \text { there are } \tilde{\mu}_{k} \in F_{\widetilde{T}}\left(\widetilde{A}_{n}\right) \backslash N \text { with } \tilde{\mu}_{k} \rightarrow \tilde{\mu} \text { weakly. }
$$

By (iii) of Lemma $4,\left(\tilde{A}_{n}, \tilde{T} \mid \widetilde{A}_{n}\right)$ decomposes into finitely many topologically transitive subshifts of finite type. Using the specification property for subshifts of finite type, it is shown in [1] that the measures concentrated on periodic orbits are dense in the set of all invariant measures on each transitive component. Since $N$ is finite and since each $\tilde{\mu} \in E_{\widetilde{T}}\left(\widetilde{A}_{n}\right)$ is concentrated on a transitive component, (3.8) follows.

We show (i). Since the discontinuities of $f$ are contained in $P \subset Q$, the functions $\tilde{f}: \tilde{A} \rightarrow(-\infty, \infty]$ and $\tilde{f}_{n}: \tilde{A} \rightarrow \mathbb{R}$ are continuous. Furthermore, (3.3) says that $\tilde{f}_{n}(x)=f_{n}(\pi(x))$, if $f_{n}$ is continuous in $\pi(x)$. Hence (3.6) 
implies that $\sup _{E_{T}\left(A_{n}, f_{n}\right)} h_{\mu}+\int f_{n} d \mu \leq \sup _{E_{\widetilde{T}}\left(\tilde{A}_{n}\right)} h_{\tilde{\mu}}+\int \tilde{f}_{n} d \tilde{\mu}$. Using (3.8), each $\tilde{\mu} \in N$ can be approximated by a sequence of $\tilde{\mu}_{k} \in F_{\widetilde{T}}\left(\tilde{A}_{n}\right) \backslash N$ and furthermore $h_{\tilde{\mu}_{k}}=0=h_{\tilde{\mu}}$. Since $f_{n}$ has its discontinuities in $P \subset Q$ for each $n$, we get $\sup _{E_{T}\left(A_{n}, f_{n}\right)} h_{\mu}+\int f_{n} d \mu \geq \sup _{E_{\tilde{T}}\left(\tilde{A}_{n}\right)} h_{\tilde{\mu}}+\int \tilde{f}_{n} d \tilde{\mu}$ by (3.7). Now (2.1) and Corollary 9.10 .1 in [14] imply $q\left(T \mid A_{n}, f_{n}\right)=p\left(\widetilde{T} \mid \tilde{A}_{n}, \tilde{f}_{n}\right)$, which together with (2.2) shows (i).

In order to show (ii) suppose that there is a $\mu \in \widetilde{E}_{T}(A, h)$ with $\int h d \mu>0$. If $\mu$ is supported on a periodic orbit in $A$, this orbit is stable because $\int h d \mu>0$. This contradicts the fact that $A$ is a Darboux subset. Hence we can suppose that $\mu$ has no atoms. By definition of $\widetilde{E}_{T}(A, h)$ there is an $n$ with $\mu \in E_{T}\left(A_{n}, h\right)$. By (3.6) there is a $\tilde{\mu} \in E_{\widetilde{T}}\left(\widetilde{A}_{n}\right)$ with $\tilde{\mu} \circ \pi^{-1}=\mu$. Since $\mu$ has no atoms, we have $\tilde{h}=h \circ \pi$ almost everywhere by (3.3) and $\int \tilde{h} d \tilde{\mu}=\int h d \mu>0$. Let $k$ be such that $\int \tilde{h}_{k} d \tilde{\mu}>0$, where $\tilde{h}_{k}=\min (\tilde{h}, k)$. By (3.8) there is a $\tilde{\nu} \in F_{\widetilde{T}}\left(\tilde{A}_{n}\right) \backslash N$ with $\int \tilde{h}_{k} d \tilde{\nu}>0$ since $\tilde{h}_{k}: \tilde{A} \rightarrow \mathbb{R}$ is continuous. Set $\nu=\tilde{\nu} \circ \pi^{-1}$, which is concentrated on a periodic orbit $L$ and is in $E_{T}\left(A_{n}, h\right)$ by (3.7). Therefore $\int h d \nu=\int \tilde{h} d \tilde{\nu} \geq \int \tilde{h}_{k} d \tilde{\nu}>0$, which implies that $L$ is a stable periodic orbit in $A_{n} \subset A$. This contradicts the fact that $A$ is a Darboux subset and (ii) is shown.

Remark. If $f$ is continuous and $\sup f<p(T \mid A, f)$ then $q(T \mid A, f)=$ $p(T \mid A, f)$, where $p(T \mid A, f)$ is defined as $\sup _{\mu \in E_{T}(A)} h_{\mu}+\int f d \mu$, if $T$ has discontinuities. This follows from results in [13] and implies $q(0)=$ $h_{\text {top }}(T \mid A)$, if $h_{\text {top }}(T \mid A)>0$.

\section{EXISTENCE OF CONFORMAL MEASURES}

In this section we show existence of conformal measures. First we consider $(\widetilde{A}, \widetilde{T} \mid \widetilde{A})$.

Lemma 7. Let $T$ be a regular piecewise monotonic map and let $A$ be a Darboux subset of $([0,1], T)$ with $h_{\text {top }}(A)>0$. Let $f \in D(A)$ and let $\tilde{f}$ be the continuous extension of $f \mid A^{\prime}$ to $\widetilde{A}$. We write $q$ for $q(T \mid A, f)$. Then there is a probability measure $\tilde{m}$ on $\tilde{A}$ such that

$$
\tilde{m}(\tilde{T}(Y))=\int_{Y} e^{q-\tilde{f}} d \tilde{m} \quad \text { if } Y \subset(\widetilde{A} \backslash \widetilde{S}) \cap U \text { for some } U \in \mathscr{U}
$$

and such that

$$
\tilde{m}(\{\tilde{T}(s)\}) \geq e^{q-\tilde{f}(s)} \tilde{m}(\{s\}) \quad \text { if } s \in \tilde{S} \cap \tilde{A} .
$$

Proof. Set $\tilde{f}_{n}=\min (n, \tilde{f})$. We write $p_{n}$ for $p\left(\tilde{T} \mid \tilde{A}_{n}, \tilde{f}_{n}\right)$. Since $\tilde{T} \mid \tilde{A}_{n}$ is an open and expansive map by (iii) of Lemma 4 and since $\tilde{f}_{n}: \tilde{A}_{n} \rightarrow \mathbb{R}$ is continuous, it follows from Theorem 3.12 of [2] that there is a probability measure $\tilde{m}_{n}$ on $\tilde{A}_{n}$ satisfying

$$
\tilde{m}_{n}(\widetilde{T}(Y))=\int_{Y} e^{p_{n}-\tilde{f}_{n}} d \tilde{m}_{n} \quad \text { if } Y \subset \widetilde{A_{n}} \cap U \text { for some } U \in \mathscr{U} \text {. }
$$

We consider $\tilde{m}_{n}$ as a measure on $X$. If $U \in \mathscr{U}_{k}$ with $k \leq n$ and $U \cap \tilde{S}=\varnothing$ then $\widetilde{T}\left(U \cap \widetilde{A}_{n}\right)=\widetilde{T}(U) \cap \widetilde{A}_{n}$ by (ii) of Lemma 4. This implies that (4.3) holds with $Y=U$. 
Now let $\tilde{m}$ be a weak limit point of the sequence $\left(\tilde{m}_{n}\right)_{n \geq 1}$. Suppose that $\left(\tilde{m}_{n_{l}}\right)_{l \geq 1}$ converges to $\tilde{m}$. In particular, $\tilde{m}$ is concentrated on $\tilde{A}$. Since $\tilde{f}: \widetilde{A} \rightarrow(-\infty, \infty]$ is continuous and since $\left(p_{n}\right)_{n \geq 1}$ converges to $q$ by (i) of Lemma 6, we get that $\left(e^{p_{n}-\tilde{f}_{n}}\right)_{n \geq 1}$ converges uniformly to $e^{q-f}$. If $U \in \mathscr{U}_{k}$ for some $k \geq 2$ then $\partial U=\varnothing$. If additionally $U \cap \widetilde{S}=\varnothing$ then also $\partial \widetilde{T}(U)=\varnothing$ by (i) of Lemma 4 and (4.3) holds with $Y=U$ for $n \geq k$. Because of $\tilde{m}_{n_{l}} \rightarrow \tilde{m}$ we get from (4.3) that

$$
\tilde{m}(\widetilde{T}(U))=\int_{U} e^{p-\tilde{f}} d \tilde{m} \text { if } U \in \bigcup_{k=2}^{\infty} \mathscr{U}_{k} \text { and } U \cap \widetilde{S}=\varnothing .
$$

The sets $U \cap \tilde{A}$ with $U \in \bigcup_{k=2}^{\infty} \mathscr{U}_{k}$ and $U \cap \tilde{S}=\varnothing$ generate the Borel sets on $\widetilde{A} \backslash \widetilde{S}$ by Lemma 3 . As $\widetilde{T}$ is strictly monotone and continuous on each element of $\mathscr{U},(4.4)$ implies (4.1), using also (ii) of Lemma 4.

In order to show (4.2) fix $s \in \widetilde{S} \cap \widetilde{A}$. Set $Y_{k}=\widetilde{A} \cap U_{k}(s)$, where $U_{k}(s)$ is the unique element of $\mathscr{U}_{k}$ which contains $s$. Now (4.3) implies

$$
\tilde{m}_{n}\left(\widetilde{T}\left(Y_{k}\right)\right) \geq \tilde{m}_{n}\left(\widetilde{T}\left(Y_{k} \cap \tilde{A}_{n}\right)\right)=\int_{Y_{k} \cap \widetilde{A}_{n}} e^{p_{n}-\tilde{f}_{n}} d \tilde{m}_{n}=\int_{Y_{k}} e^{p_{n}-\tilde{f}_{n}} d \tilde{m}_{n} .
$$

Since $\partial Y_{k}=\varnothing$, since $e^{p_{n}-\tilde{f}_{n}}$ converges uniformly to $e^{q-\tilde{f}}$, and since $\widetilde{T}\left(Y_{k}\right)$ is closed, $\tilde{m}_{n_{l}} \rightarrow \tilde{m}$ implies

$$
\tilde{m}\left(\tilde{T}\left(Y_{k}\right)\right) \geq \int_{Y_{k}} e^{q-\tilde{f}} d \tilde{m} .
$$

By Lemma 3 we have $\bigcap_{k=1}^{\infty} Y_{k}=\{s\}$ and (4.2) follows with $k \rightarrow \infty$.

Lemma 8. Let $T$ be a regular piecewise monotonic map, let $A$ be a Darboux subset of $([0,1], T)$ and let $f \in D(A)$. Let $\tilde{m}$ be a measure on $\tilde{A}$ satisfying (4.1). If $\widetilde{A}$ is topologically transitive, then $\tilde{m}$ is positive on open subsets of $\tilde{A}$.

Proof. In the notation of [8], (i) of Lemma 5 says that $(\tilde{A}, \widetilde{T} \mid \tilde{A})$ is a piecewise monotonic dynamical system. Let $I$ be an open interval in $\widetilde{A}$. Since $\widetilde{A}$ is topologically transitive, Lemma 1 of [8] implies that $\bigcup_{i=0}^{r} \widetilde{T}^{i}(I)=\widetilde{A}$ for some $r$. Suppose that $\tilde{m}(I)=0$. By (4.1) we get that $\tilde{m}\left(\tilde{T}^{i}(I) \backslash \bigcup_{j=1}^{i} \tilde{T}^{j}(\tilde{S})\right)=0$ for $i \geq 1$. Hence $\tilde{m}$ is concentrated on $\bigcup_{j=1}^{r} \widetilde{T}^{j}(\widetilde{S})$. Since $\widetilde{T}^{j}(\widetilde{S}) \cap \widetilde{Q}=\varnothing$ for $j \geq 1$ we have $e^{p-\tilde{f}}>0$ on $\bigcup_{j=1}^{\infty} \widetilde{T}^{j}(\widetilde{S})$, since $f \in D(A)$. Hence, for $x \in \bigcup_{j=1}^{\infty} \widetilde{T}^{j}(\widetilde{S})$, we get from $(4.1)$, that $\tilde{m}(\{x\})>0$ implies $\tilde{m}(\{\tilde{T}(x)\})>0$. Since the orbits of points in $\widetilde{S}$ are infinite, $\tilde{m}$ cannot be concentrated on $\bigcup_{j=1}^{r} \widetilde{T}^{j}(\widetilde{S})$. Therefore $\tilde{m}(I)=0$ is impossible.

Now we go back to $A$. We say that $(A, T \mid A)$ is topologically transitive, if it contains a dense orbit, which is disjoint from the set $P$ of partition points.

Theorem 2. Let $T$ be a regular piecewise monotonic map and let $A$ be a Darboux subset of $([0,1], T)$ with $h_{\text {top }}(A)>0$. If $f \in D(A)$, then there exists an almost $e^{q(T \mid A, f)-f}$-conformal measure $m$ on $A$. If $(A, T \mid A)$ is topologically transitive, then there is such an $m$, which is positive on open subsets of $A$. 
Proof. Let $\tilde{m}$ be as in Lemma 7 and set $m=\tilde{m} \circ \pi^{-1}$. Since $\pi(\tilde{A})=A, m$ is a measure on $A$. We write $q$ for $q(T \mid A, f)$. If $Y \subset A \cap Z$ for some $Z \in \mathscr{Z}$ then (3.4) implies that $\pi^{-1}(Y) \cap \widetilde{S}=\varnothing$ and hence $\pi^{-1}(Y)$ is a finite union of disjoint sets, each of which is contained in $(\widetilde{A \backslash} \backslash \widetilde{S}) \cap U$ for some $U \in \mathscr{U}$. Furthermore $\widetilde{T}$ is strictly monotone on $\pi^{-1}(Y)$, the definition of $\pi$ and (3.4) imply $\pi^{-1}(T(Y))=\tilde{T}\left(\pi^{-1}(Y)\right)$ which gives $m(T(Y))=\tilde{m}\left(\tilde{T}\left(\pi^{-1}(Y)\right)\right)$, and $\int_{Y} e^{q-f} d m=\int_{\pi^{-1}(Y)} e^{q-\tilde{f}} d \tilde{m}$ by (3.3) since discontinuities of $f$ are contained in $P$. Using these facts one sees that (1.1) follows from (4.1) showing that $m$ is an almost $e^{q-f}$-conformal measure.

If $(A, T \mid A)$ is topologically transitive, it follows from $\pi(\widetilde{A})=A$, that also $(\tilde{A}, \tilde{T} \mid \widetilde{A})$ is topologically transitive. Lemma 8 implies that $\tilde{m}$ is positive on open sets in $\tilde{A}$. This implies that the same holds for $m=\tilde{m} \circ \pi^{-1}$ on $A$ finishing the proof.

In order to explain our construction consider the following example. Define $T:[-1,1] \rightarrow[-1,1]$ by $T(x)=-|\sin 4 x|$ if $x \in[0,1]$ and by $T(x)=$ $|\sin 4 x|$ if $x \in[-1,0]$. We have $P=\left\{-1,-\frac{\pi}{4},-\frac{\pi}{8}, \frac{\pi}{8}, \frac{\pi}{4}, 1\right\}$. Let $g$ be a function on $[-1,1]$ with $g(0)=1$. Then the measure $\delta$ concentrated on $\{0\}$ is an almost $g$-conformal measure. This follows since $T^{-1}(\{0\}) \subset P$ and since $\delta(\{T(0)\})=g(0) \delta(\{0\})$. Although $A=[-1,1]$ is a topologically transitive set, this measure $\delta$ is supported on a single point. Such a measure is useless for applications. In order to exclude such degenerate cases and to get Theorem 2, the approximation procedure of $\S \S 2$ and 3 leading to the definition of $q(T \mid A, f)$ and via Lemma 7 to Theorem 2 is chosen. The method of [4], where it was sufficient to cut out only neighbourhoods of $P$, would not exclude such degenerate cases.

Finally we give conditions under which conformal measures exist. The next theorem is a generalization of Theorem 1 in [9].

Theorem 3. Let $T$ be a regular piecewise monotonic map and let $A$ be a Darboux subset of $([0,1], T)$ with $h_{\text {top }}(A)>0$. If $f \in D(A)$ satisfies $\sup f<$ $q(T \mid A, f)$ then there is an atomfree $e^{q(T \mid A, f)-f}$-conformal measure $m$ on $A$. If $A$ is topologically transitive, then $m$ is positive on open subsets of $A$.

Proof. Let $\tilde{m}$ be as in Lemma 7 and set $m=\tilde{m} \circ \pi^{-1}$. Set $q=q(T \mid A, f)$ and $\alpha=q-\sup f>0$. For $x \in \tilde{A}$ it follows from (4.1) and (4.2) that $\tilde{m}(\{\tilde{T}(x)\}) \geq e^{\alpha} \tilde{m}(\{x\})$. Since $e^{\alpha}>1$, one gets $\tilde{m}\left(\left\{\tilde{T}^{i}(x)\right\}\right)>1$ for some $i$, if $m(\{x\})>0$. Hence $\tilde{m}$ and hence also $m$ have no atoms. This implies that (4.1) holds for all $Y \subset \tilde{A} \cap U$ with $U \in \mathscr{U}$ and that (1.1) holds for $Y \subset A$, for which $T \mid Y$ is monotone, showing that $m$ is $e^{q-f}$-conformal. The last statement follows as in the proof of Theorem 2 .

\section{DyNAMiCAL DIMENSION}

In this section we investigate the dynamical dimension $\operatorname{dD}(A)$ of $A$ using conformal measures. Here almost $\left|T^{\prime}\right|^{t}$-conformal measures are of interest, which we shall also call almost $t$-conformal. Hence a probability measure $m$ on $A$ is almost $t$-conformal if 


$$
m(T(Y))=\int_{Y}\left|T^{\prime}\right|^{t} d m \text { for all } Y \subset A \text { contained in some } Z \in \mathscr{Z} \text {. }
$$

Let $\delta(A)$ be the infimum of all $t$ for which an almost $t$-conformal measure exists on $A$, which is positive on open subsets of $A$. We want to compare the dynamical dimension $\mathrm{dD}(A)$ defined in the introduction, the minimal zero $z_{A}$ of $t \mapsto q(t)$ introduced in $\S 2$, and $\delta(A)$. In order to apply results of [10] we define $p$-variation for $p>0$. For $g:[0,1] \rightarrow \mathbb{R}$ and $I$ a subinterval of $[0,1]$ define

$$
\operatorname{var}_{I}^{p} g=\sup \left\{\sum_{i=1}^{m}\left|g\left(x_{i-1}\right)-g\left(x_{i}\right)\right|^{p}: m \in \mathbb{N}, x_{i} \in I, x_{0}<x_{1}<\cdots<x_{m}\right\} .
$$

Now we can show

Lemma 9. Let $T$ be regular and piecewise differentiable with $T^{\prime} \mid Z \neq 0$ and $\operatorname{var}_{Z}^{p} T^{\prime}<\infty$ for all $Z \in \mathscr{Z}$ and some $p>0$. Let $A$ be a Darboux subset of $([0,1], T)$ with $h_{\mathrm{top}}(T \mid A)>0$. Then $\mathrm{dD}(A) \geq z_{A}$.

Proof. The assumptions on $T^{\prime}$ imply that $h:=-\log \left|T^{\prime}\right|$ is in $D(A)$. Theorem 1 shows the existence of $z_{A}$. Nothing is to show if $z_{A}=0$. Hence suppose that $z_{A}>0$. Choose $t \in\left(0, z_{A}\right)$ arbitrary. Then $q(t)>0$. By Lemma 1 there is a $\mu \in \widetilde{E}_{T}(A, h)$ with $h_{\mu}+t \int h d \mu>0$ and $h_{\mu}+z_{A} \int h d \mu \leq 0$. This implies $\left(z_{A}-t\right) \int h d \mu<0$ and $\chi_{\mu}:=-\int h d \mu>0$. Together with $\operatorname{var}_{Z}^{p} T^{\prime}<\infty$ for all $Z \in \mathscr{Z}$ this gives the assumptions of Theorem 1 in [10], which says that $\operatorname{HD}(\mu)=h_{\mu} / \chi_{\mu}$. Since $h_{\mu}+t \int h d \mu>0$, we get $\operatorname{HD}(\mu)>t$ and $h_{\mu}>0$. Therefore $\operatorname{dD}(A) \geq \operatorname{HD}(\mu)>t$. Since $t \in\left(0, z_{A}\right)$ was arbitrary, we get $\mathrm{dD}(A) \geq z_{A}$.

Lemma 10. Let $T$ be regular and piecewise differentiable with $T^{\prime} \mid Z \neq 0$ for all $Z \in \mathscr{Z}$. Let $A$ be a Darboux subset of $([0,1], T)$ with $h_{\mathrm{top}}(T \mid A)>0$, which is topologically transitive. Then $z_{A} \geq \delta(A)$.

Proof. The assumptions on $T^{\prime}$ imply that $h:=-\log \left|T^{\prime}\right|$ is in $D(A)$. Theorem 1 implies that $q\left(T \mid A, z_{A} h\right)=q\left(z_{A}\right)=0$. Now Theorem 2 implies the existence of an almost $z_{A}$-conformal measure on $A$, which is positive on open subsets, since $A$ is topologically transitive. Hence $\delta(A) \leq z_{A}$.

Lemma 11. Let $T$ be regular and piecewise differentiable with $T^{\prime} \mid Z \neq 0$ and $\operatorname{var}_{Z}^{p} T^{\prime}<\infty$ for all $Z \in \mathscr{Z}$ and some $p>0$. Let $A$ be a Darboux subset of $([0,1], T)$ with $h_{\text {top }}(T \mid A)>0$. Then $\delta(A) \geq \mathrm{dD}(A)$.

Proof. Suppose that $\delta(A)<\mathrm{dD}(A)$. By definition of $\mathrm{dD}(A)$ there is a $\mu \in$ $E_{T}(A)$ with $h_{\mu}>0$ and $\delta(A)<\operatorname{HD}(\mu)$. By definition of $\delta(A)$ there is a $t \in[\delta(A), \operatorname{HD}(\mu))$ such that an almost $t$-conformal measure $m$ exists on $A$, which is positive on open subsets. Set $h=-\log \left|T^{\prime}\right|$ and $\chi_{\mu}=-\int h d \mu$. Since $T$ is piecewise differentiable, it follows from Theorem 2 in [7] that $\chi_{\mu}>0$, as $h_{\mu}>0$. Fix now $s \in(t, \operatorname{HD}(\mu))$ and $\varepsilon \in\left(0, \chi_{\mu} / 2\right)$ such that $s-t \geq$ $2 \varepsilon t /\left(\chi_{\mu}-2 \varepsilon\right)$. By the assumptions on $T^{\prime}$ the function $g$ defined by $g=T^{\prime}$ on $[0,1] \backslash P$ and $g=0$ on $P$ satisfies $\operatorname{var}_{[0,1]}^{p} g<\infty$. Since $\mu$ is ergodic and since $h_{\mu}>0$ holds, $\mu$ has no atoms and hence $\mu\left(\bigcup_{i=0}^{\infty} T^{-i}(P)\right)=0$. 
Furthermore we have $h \in L_{\mu}^{1}$, since $\chi_{\mu}=-\int h d \mu>0$ and since $\inf h>-\infty$, as $T^{\prime}$ is bounded. We have checked the assumptions of Lemma 1 in [10] (see also Lemma 1 in [7]), which implies that there exists a finite or countable family $\mathscr{Y}$ of open pairwise disjoint intervals refining $\mathscr{Z}$, such that

$$
\begin{gathered}
\mu\left(\bigcup_{Y \in \mathscr{Y}} Y\right)=1, \\
\sup _{x, y \in Y}|h(x)-h(y)| \leq \varepsilon \quad \text { for all } Y \in \mathscr{Y}, \\
-\sum_{Y \in \mathscr{Y}} \mu(Y) \log \mu(Y)<\infty .
\end{gathered}
$$

Set $\mathscr{Y}_{n}=\bigvee_{i=0}^{n-1} T^{-i \mathscr{Y}}$ and $L=\bigcap_{i=0}^{\infty} T^{-i}\left(\bigcup_{Y \in \mathscr{Y}} Y\right)=\bigcap_{n=0}^{\infty} \bigcup_{Y \in \mathscr{Y} n} Y$, which has $\mu$-measure one by (5.2). For $x \in L$ let $Y_{n}(x)$ be the unique element of $\mathscr{Y}_{n}$ which contains $x$. Using the technique of lifting $\mu$ to the Markov extension of $([0,1], T)$ over $\mathscr{Y}$, which is possible by $(5.4)$ and $h_{\mu}>0$, it is shown in $\S 4$ of [10] (see also Lemma 2 of [7]) that there is a nonempty open interval $J$ with the following property. For $\mu$-almost every $x \in L$ there is an infinite set $I(x) \subset \mathbb{N}$ with

$$
J=T^{n}\left(Y_{n+1}(x)\right) \subset T^{n}\left(Y_{n}(x)\right) \text { for } n \in I(x) .
$$

Since there is an $x \in L \cap A$ and an $n$ with $J=T^{n}\left(Y_{n+1}(x)\right)$, we have $T^{n}(x) \in$ $J$ and $J \cap A \neq \varnothing$. As $m$ is positive on open subsets of $A$, we get $m(J)=$ $m(J \cap A)>0$. Since $T^{i}\left(Y_{n}(x)\right)$ is contained in some element of $\mathscr{Y}$ and hence in some element of $\mathscr{Z}$ for $0 \leq i \leq n-1$, we get from (5.1) that $m\left(T^{i}\left(Y_{n}(x)\right)\right) \geq$ $\inf _{T^{i}\left(Y_{n}(x)\right)}\left|T^{\prime}\right|^{-t} m\left(T^{i+1}\left(Y_{n}(x)\right)\right)$. Furthermore $\inf _{T^{i}\left(Y_{n}(x)\right)}\left|T^{\prime}\right|^{-t}=\inf _{T^{i}\left(Y_{n}(x)\right)} e^{t h}$ $\geq e^{t h\left(T^{i}(x)\right)-t \varepsilon}$ by (5.3). Together with (5.5) we get for $\mu$-almost all $x$

$$
m\left(Y_{n}(x)\right) \geq m(J) e^{t S_{n} h(x)-n t \varepsilon} \quad \text { if } n \in I(x)
$$

where $S_{n} h(x)=\sum_{i=0}^{n-1} h\left(T^{i}(x)\right)$. Let $|I|$ denote the length of the interval $I$. In the same way as (5.6) we get using the mean value theorem and again (5.3) that

$$
\left|Y_{n}(x)\right| \leq e^{S_{n} h(x)+n \varepsilon} \quad \text { for } x \in L \text { and } n \geq 1
$$

as $\left|T^{n}\left(Y_{n}(x)\right)\right| \leq 1$. Since $\mu$ is ergodic and since $\chi_{\mu}=-\int h d \mu$, we get $\lim _{n \rightarrow \infty} \frac{1}{n} S_{n} h(x)=-\chi_{\mu}$ for $\mu$-almost all $x$ by the ergodic theorem. Hence (5.7) implies that for $\mu$-almost all $x$ there is an $n(x)$ with

$$
\left|Y_{n}(x)\right| \leq e^{-n x_{\mu}+2 n \varepsilon} \quad \text { for all } n \geq n(x) .
$$

Now let $M \subset L$ be the set of all $x$ for which (5.6) and (5.8) hold. Then $\mu(M)=1$. Fix $x \in M$ and $n \in I(x)$ with $n \geq n(x)$. Then we have

$$
\begin{aligned}
\frac{\left|Y_{n}(x)\right|^{s}}{m\left(Y_{n}(x)\right)} & \leq \frac{\left|Y_{n}(x)\right|^{t}}{m\left(Y_{n}(x)\right)}\left|Y_{n}(x)\right|^{s-t} \\
& \leq \frac{1}{m(J)} e^{2 n t \varepsilon} e^{-n\left(x_{\mu}-2 \varepsilon\right)(s-t)} \quad \text { by }(5.6),(5.7) \text { and }(5.8) \\
& \leq \frac{1}{m(J)} \quad \text { by the choice of } \varepsilon \\
& =: c<\infty \quad \text { since } m(J)>0 .
\end{aligned}
$$


Now fix $\delta>0$ arbitrary. For $x \in M$ there is an $n$ such that $R(x):=Y_{n}(x)$ satisfies $|R(x)|<\delta$ and $|R(x)|^{s} \leq c m(R(x))$ using (5.8) and the above estimate. Since two elements of $\bigcup_{n=1}^{\infty} \mathscr{Y}_{n}$ are either disjoint or one contains the other, there is a subset $\mathscr{R}$ of $\{R(x): x \in M\}$ which consists of pairwise disjoint intervals and which covers $M$. We get $\sum_{R \in \mathscr{R}}|R|^{s} \leq c \sum_{R \in \mathscr{R}} m(R) \leq c$. It follows now from the definition of the $s$-dimensional Hausdorff measure $\nu_{s}$ (see [5]), that $\nu_{s}(M) \leq c$. This implies that the Hausdorff dimension $\operatorname{HD}(M)$ of $M$ satisfies $\operatorname{HD}(M) \leq s$. Since $\mu(M)=1$, we get $\operatorname{HD}(\mu) \leq s$, which contradicts the choice of $S$. Hence $\delta(A) \geq \mathrm{dD}(A)$.

We collect the results of the above three lemmas in the following theorem.

Theorem 5. Let $T$ be regular and piecewise differentiable with $T^{\prime} \mid Z \neq 0$ and $\operatorname{var}_{Z}^{p} T^{\prime}<\infty$ for all $Z \in \mathscr{Z}$ and some $p>0$. Let $A$ be a Darboux subset of $([0,1], T)$ with $h_{\text {top }}(T \mid A)>0$, which is topologically transitive. Then $\operatorname{dD}(A)=$ $z_{A}=\delta(A)$.

Remark. In [8] for a larger class of functions than $D(A)$ a pressure $p(T \mid A, f)$ is defined, for which the variational principle holds. Therefore it coincides with the usual pressure, if $T \mid A$ and $f$ are continuous (see [14]). Set $p(t)=$ $p(T \mid A, t h)$, where $h:=-\log \left|T^{\prime}\right| \in D(A)$. The problem here is to show $p(1) \leq$ 0 (Theorem 1 of [8] proves that this holds, if $T$ is continuous or if $\inf \left|T^{\prime}\right| \geq 1$, but in general this question is open), which gives the existence of

$$
y_{A}:=\inf \{t: p(t)=0\} .
$$

We assume that $y_{A}$ exists. Because of $q(T \mid A, f) \leq p(T \mid A, f)$, which follows from (2.1), we get $z_{A} \leq y_{A}$. Furthermore, the proof of Theorem 2 in [8], which is essentially the same as that of Lemma 9 , shows that $y_{A} \leq \mathrm{dD}(A)$. Therefore, under the assumptions of Theorem 5, we get $y_{A}=z_{A}$, provided that $y_{A}$ exists.

\section{REFERENCES}

1. M. Denker, C. Grillenberger, and K. Sigmund, Ergodic theory on compact spaces, Lecture Notes in Math., vol. 527, Springer-Verlag, Berlin, Heidelberg, and New York, 1976.

2. M. Denker and M. Urbański, On the existence of conformal measures, Trans. Amer. Math. Soc. 328 (1991), 563-587.

3. _ Hausdorff and conformal measures on Julia sets with a rationally indifferent periodic point, J. London Math. Soc. 43 (1991), 107-118.

4. __ On Sullivan's conformal measures for rational maps of the Riemann sphere, Nonlinearity 4 (1991), 365-384.

5. K. J. Falconer, The geometry of fractal sets, Cambridge Univ. Press, 1985.

6. F. Hofbauer, Piecewise invertible dynamical systems, Probab. Theory Related Fields 72 (1986), 359-386.

7. __ An inequality for the Ljapunov exponent of an ergodic invariant measure for a piecewise monotonic map of the interval, Lyapunov Exponents (L. Arnold, H. Crauel, and J.-P. Eckmann, eds.), Lecture Notes in Math., vol. 1486, Springer-Verlag, Berlin and Heidelberg, 1991, pp. 227-231.

8. __ Hausdorff dimension and pressure for piecewise monotonic maps of the interval, $\mathbf{J}$. London Math. Soc. (to appear).

9. _ـ Hausdorff and conformal measures for expanding piecewise monotonic maps of the interval, Studia Math. 103 (1992), 191-206. 
10. F. Hofbauer and P. Raith, The Hausdorff dimension of an ergodic invariant measure for a piecewise monotonic map of the interval, Canad. Math. Bull 35 (1991), 1-15.

11. M. Martens, W. de Melo, and S. van Strien, Julia-Fatou-Sullivan theory for real one-dimensional dynamics, Acta Math. 168 (1993), 273-318.

12. W. de Melo and S. van Strien, One-dimensional dynamics, Springer-Verlag, 1993.

13. P. Raith, Hausdorf dimension for piecewise monotonic maps, Studia Math. 94 (1989), 17-33.

14. P. Walters, An introduction to ergodic theory, Springer-Verlag, Berlin, Heidelberg, and New York, 1982.

Institut für Mathematik, Universität Wien, Strudlhofgasse 4, A-1090 Wien, Austria

E-mail address: a8131dar@awiuni11.bitnet and a81131dar@awiuni11.edvz.univie. ac. at

Department of Mathematics, University of North Texas, Denton, Texas 76203-5116

E-mail address: mariuszu@vaxb.acs . unt.edu 